\title{
Quantitative approach to skin field cancerization using a nanoencapsulated photodynamic therapy agent: a pilot study
}

This article was published in the following Dove Press journal:

Clinical, Cosmetic and Investigational Dermatology

16 February 2013

Number of times this article has been viewed

\author{
Simone K Passos ${ }^{1,2}$ \\ Paulo EN de Souza ${ }^{3}$ \\ Priscila KP Soares ${ }^{1,3}$ \\ Danglades RM Eid ${ }^{1,2}$ \\ Fernando L Primo ${ }^{4}$ \\ Antonio Cláudio Tedesco ${ }^{4}$ \\ Zulmira GM Lacava' \\ Paulo C Morais ${ }^{3,5}$ \\ 'University of Brasília, Institute \\ of Biological Sciences, DF, Brazil; \\ ${ }^{2}$ Foundation for Teaching and \\ Research on Health Sciences, Brasília,

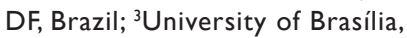 \\ Institute of Physics, Brasília, DF, Brazil; \\ ${ }^{4}$ Department of Chemistry, Faculty \\ of Philosophy, Sciences and Letters \\ of Ribeirão Preto, Laboratory of \\ Photobiology and Photomedicine, \\ University of São Paulo, Ribeirão \\ Preto, São Paulo, Brazil; ${ }^{5}$ Department \\ of Control Science and Engineering, \\ Hua-Zhong University of Science and \\ Technology, Wuham, People's Republic \\ of China
}

Correspondence: Zulmira GM Lacava Institute of Biological Sciences, University of Brasília, 70-910-900 Brazil

Tel +55 6I 31073087

Fax+556131073087

Email zulmira@unb.br
Background: This paper introduces a new nanoformulation of 5-aminolevulinic acid (nano-ALA) as well as a novel quantitative approach towards evaluating field cancerization for actinic keratosis and/or skin photodamage. In this pilot study, we evaluated field cancerization using nano-ALA and methyl aminolevulinate (MAL), the latter being commercialized as Metvix $^{\circledR}$.

Methods and results: Photodynamic therapy was used for the treatment of patients with selected skin lesions, whereas the fluorescence of the corresponding photosensitizer was used to evaluate the time evolution of field cancerization in a quantitative way. Field cancerization was quantified using newly developed color image segmentation software. Using photodynamic therapy as the precancer skin treatment and the approach introduced herein for evaluation of fluorescent area, we found that the half-life of field cancerization reduction was 43.3 days and 34.3 days for nano-ALA and MAL, respectively. We also found that nano-ALA targeted about $45 \%$ more skin lesion areas than MAL. Further, we found the mean reduction in area of skin field cancerization was about $10 \%$ greater for nano-ALA than for MAL.

Conclusion: Although preliminary, our findings indicate that the efficacy of nano-ALA in treating skin field cancerization is higher than that of MAL.

Keywords: photodynamic therapy (PDT), nanoemulsion, 5-aminolevulinic acid, actinic keratosis, field cancerization, skin cancer

\section{Introduction}

Nowadays, the term "field cancerization" is used broadly to "mean the process whereby cells in a particular tissue or organ are transformed, such that genetically altered but histologically normal-appearing cells predate the development of neoplastic disease or coexist with malignant cells." Ultimately, field cancerization is related to cells with cancer-associated genetic alterations but without invasive growth. ${ }^{1}$ Field cancerization has been reported on almost all epithelial surfaces, in particular on the skin, which is the organ most exposed to environmental carcinogens, such as ultraviolet radiation, ${ }^{1,2}$ and also to other skin disorders that can evolve to a skin cancer. ${ }^{3}$ The preneoplastic phase in skin likely reflects accumulation of mutations ${ }^{1}$ that form the basis for the carcinogenesis process. ${ }^{1,4-6}$ The literature emphasizes that if a tumor is excised from a predisposed epidermis, recurrence may occur by neoplastic conversion of residual parts of field cancerization. ${ }^{6}$ Furthermore, it has been widely reported that after a first tumor occurrence, the chance of recurrence is statistically enhanced. ${ }^{7}$

The most common skin lesion caused by ultraviolet $\mathrm{B}$ radiation is actinic keratosis, which is considered the precursor lesion of squamous cell carcinoma. Actinic keratosis 
and invasive squamous cell carcinoma share histopathological features, and both present genetic tumor markers and p53 mutation. ${ }^{8}$ However, there is accumulating evidence that actinic keratosis and squamous cell carcinoma are part of a broad spectrum, running from sun-damaged skin to squamous cell carcinoma, with both demonstrating alterations in the p53 gene, the signature of early skin carcinogenesis. Studies reveal a direct correlation between abnormal gene expression and progression from normal skin to photodamaged skin and from actinic keratosis to squamous cell carcinoma., ${ }^{9,10}$ Actinic keratosis has been classified as early squamous cell carcinoma in situ type I, II, and III. ${ }^{11-13}$ An estimated $12 \%-13 \%$ of patients with untreated actinic keratosis may develop invasive squamous cell carcinoma. ${ }^{14}$ Organ transplant recipients are particularly and severely affected by skin-related field cancerization; within 5 years of transplant, about $40 \%$ of organ transplant recipients develop actinic keratosis and have a 40-250-fold increase in occurrence of squamous cell carcinoma, with a 10-fold increase in mortality due to squamous cell carcinoma. ${ }^{15}$ Current international guidelines recommend treatment of actinic keratoses to avoid the potential for their development into squamous cell carcinomas. ${ }^{14,16-18}$ Exposure to ultraviolet B radiation, which indeed triggers molecular changes, precedes the appearance of actinic keratosis, and these lesions can be diagnosed at the subclinical stage using molecular techniques, although these methods would be difficult to implement on a large scale for routine screening. However, the fluorescence recorded from photosensitizing agents used in photodynamic therapy (PDT) can be used qualitatively to assess for the presence of field cancerization in the skin, ${ }^{19}$ thus offering a great window of opportunity in the routine hospital setting for the diagnosis and treatment of field cancerization in the preclinical phase. Nevertheless, a protocol for quantitative evaluation of the extent of field cancerization using the fluorescence of photosensitizers has not been devised as yet. Difficulty in assessing such information persists, leading to naïve control of efficacy of the cancer therapy and prevention approaches used.

PDT has been used worldwide for treatment of field cancerization, being very effective for actinic keratosis, while employing 5-aminolevulinic acid (ALA) or its derivative methyl aminolevulinate (MAL) as photosensitizing precursors. ${ }^{8,19-22}$ In short, PDT is based on activation of a photosensitizing precursor by visible light to produce cytotoxic oxygen species in the presence of oxygen contained in cells or tissues, that ultimately promote cell death. ${ }^{22}$ For treatment of actinic keratosis, the photosensitizing precursor is applied over the skin lesion and, after a period of occlusion, the photosensitizing precursor (ie, ALA or MAL) is converted into photoactivatable porphyrins, in particular protoporphyrin IX, with preferential accumulation in neoplastic or dysplastic tissues. ${ }^{23}$ After the period of occlusion, application of visible light triggers production of activated (cytotoxic) oxygen species, thereby leading to cell damage and consequent cell death. The safety and efficacy of topical ALA and MAL for PDT have been fully demonstrated in the treatment of actinic keratoses. ${ }^{18,23-30}$ Nevertheless, the instability of ALA in aqueous formulation and its low lipid solubility, the latter limiting the ability to penetrate through skin or cell membranes, have been identified as the main drawback of this photosensitizing precursor, thus restricting its use in PDT to superficial disease. ${ }^{31}$ An approach to overcome the drawbacks of ALA was the introduction of more lipophilic derivatives, eg, the MAL photosensitizing precursor. MAL was approved for PDT, not only for superficial lesions but also for the treatment of small nodular carcinomas due to its superior skin penetration compared with ALA. ${ }^{16,19}$ However, no significant difference in efficacy for treatment of actinic keratosis was demonstrated between ALA + PDT and MAL + PDT in a comparative study. ${ }^{32}$ MAL (Metvix ${ }^{\circledR}$ Photocure ASA, Oslo, Norway) is currently approved and commercialized in many countries (eg, US, Europe, and Brazil) whereas ALA is commercialized only in the US (Levulan ${ }^{\circledR}$, Kerastic Dusa Pharmaceuticals Inc, Wilmington, MA).

The recent worldwide trend of nanoencapsulation of drugs has raised the question of whether a nanophotosensitizing precursor would be more efficient than a free photosensitizing precursor for evaluation, prevention, and treatment for field cancerization of actinic keratoses. In order to explore the outcomes of nanoencapsulation of photosensitizing precursors in more depth, ALA was selected for encapsulation in a nanoemulsion, whereas MAL was used as purchased on the market. At this point, it is important to emphasize that the literature has reported many comparative studies involving both free ALA and free MAL. Several advantages of MAL over ALA have been reported, including a higher permeation profile while crossing the stratum corneum and cell membranes, higher selectivity (eg, in solar actinic keratoses), increased formation of protoporphyrin IX leading to higher fluorescence and photodynamic efficiency, less pain, fewer systemic effects after local treatment, and faster clearance from cells and tissues. ${ }^{33}$ For instance, a noduloulcerative basal cell carcinoma could not be sufficiently penetrated by ALA. ${ }^{26}$ It was demonstrated that the fluorescence emitted from a skin 
lesion when using ALA was less specific and less intense than when using MAL. Further, the occlusion time for ALA varied from 4 to 8 hours, whereas just 3 hours were needed for MAL. On the other hand, it was shown that ALA was more effective than MAL for the treatment of inflammatory lesions. ${ }^{19}$ However, in vitro experiments required a higher concentration of MAL than ALA for efficient formation of protoporphyrin IX.$^{33,34}$ Indeed, the superiority of MAL over ALA is still an open question and many aspects need to be evaluated. ${ }^{35}$

The aim of the present study was to carry out a pilot trial which included development of an efficient, robust, and inexpensive protocol for quantifying the time evolution of field cancerization for actinic keratosis using a combination of PDT, within a material platform of nanoencapsulation of a commercial photosensitizing agent, plus a new-developed software for photoimaging evaluation of as-treated field cancerization areas, by color image segmentation ${ }^{36}$ using a new image filter and software especially developed for this purpose. The present study of field cancerization in actinic keratosis reports the outcome of PDT using the nanoencapsulated photosensitizer (nano-ALA) compared with MAL in promoting changes in the half-life of reduction in field cancerization, extent of the targeted area of field cancerization, and effectiveness for fractional reduction in the area of field cancerization. This study also demonstrates the robustness of the as-developed software for photoimaging evaluation of field cancerization, while providing a very effective and noninvasive tool for prevention and follow-up on a clinical basis.

\section{Materials and methods}

Two photosensitizers were used in the present study, ie, nano-ALA and MAL. Whereas commercial Metvix ${ }^{\circledR}$ incorporates $16.8 \%$ of MAL in its formulation, the as-developed nano-ALA was elaborated with $20 \%$ ALA. Nano-ALA encapsulates its photosensitizing agent onto a polymericbased nanoemulsion produced from polylactide-polyglycol and egg-phosphatidylcholine lipids (50:50), with an average size ranging from $100 \mathrm{~nm}$ to $300 \mathrm{~nm}$. The oil-in-water $(\mathrm{o} / \mathrm{w})$ polymeric nanoemulsion was prepared in the presence of ALA by a spontaneous emulsification process, as described in the literature.$^{37}$ In brief, the organic phase (acetone or mixture of organic solvents) containing medium-chain triglycerides, natural soy phospholipids, and ALA (Dye Pharmaceuticals, São José dos Campos, São Paulo, Brazil, a final concentration of $20 \%$ ) was prepared at $55^{\circ} \mathrm{C}$. Subsequently, this organic solution was added into the aqueous phase containing the anionic surfactant, poloxamer 188 (Sigma Aldrich, St Louis,
MO, USA), under magnetic stirring. The organic solvent was removed by evaporation under reduced pressure at $60^{\circ} \mathrm{C}$ for 6 hours. Formulations without the photosensitizing agent were prepared under the same conditions to be used as a reference for spectroscopic and photobiologic analyses.

This blinded-observer, intraindividual pilot study was carried out in three patients clinically diagnosed with bilateral field cancerization associated to actinic keratosis and/or photodamage on the face. Pre-existing clinically diagnosed lesions suspicious for basal cell carcinoma, Bowen's disease, or invasive squamous cell carcinoma were biopsied and treated appropriately within the protocols of the Skin Cancer Service at the Public Hospital, Brasília, Brazil. Patients with hypersensitivity to ALA or porphyrins, those undergoing immunosuppressive therapy, those suffering from porphyria, and those receiving systemically acting drugs with phototoxicity or photoallergic potential were excluded from participation in the study. To minimize the effects of chronic sun exposure that vary by side of the body depending on individual habits, eg, being a car driver or a passenger, and its possible consequences for determining the relative efficacy of nano-ALA and MAL, the three patients participating in this study were confirmed to have no particular sun exposure habits.

The patients selected for the study were booked for PDT and underwent a sequence of four PDT sessions, scheduled 3-4 weeks apart. Treatment options, risks, and the likelihood of success were fully explained to the patients, who agreed to undergo the PDT protocol, and read, discussed, and signed the consent form, which is part of the document file approved by the hospital's human ethics committee.

Prior to PDT, the patients' faces were cleaned with alcohol. A thin layer of the photosensitizing percursor (nano-ALA and MAL) was accurately applied on each patient's hemiface. The treated areas on both sides of the face were occluded using a plastic film as the first layer, followed by aluminum thin foil as the top layer. To treat the field cancerization, the appropriate occlusion time was established as being 2 hours. ${ }^{38}$ After the occlusion period, the treated areas were uncovered and cleaned with gauze and physiologic serum. A Wood's lamp (model LY - 6MWD, Yoshi, São Paulo, Brazil) was then used to illuminate both sides of the treated faces while images of the fluorescent areas were digitally recorded using a Lumix-Panasonic ${ }^{\circledR}$ camera (model DMC-ZS1-12x. Osaka, Japan) in high exposure mode (flash off). The pictures were acquired at a fixed distance of $10 \mathrm{~cm}$ from the patient's face. In order to achieve the highest fluorescence image quality while recording the images, all light sources in the room except the Wood's lamp were turned off or covered with dark 
material. In our analysis, a slight red (soft pink) fluorescence was established as the minimal fluorescence signal. The fluorescence was evaluated inside a chosen polygon connecting the angle of the mandible, the inner and outer corners of the eye, and the lip commissure.

Following this image recording step, the treated areas were illuminated for 10 minutes with a light-emitting diode (LED) source (Multiwaves ${ }^{\circledR}$, Indústria Mecânica Fina, São Paulo, Brazil) tuned to a wavelength of $630 \mathrm{~nm}$ (Red-Spot) and set at $11 \mathrm{~mW} / \mathrm{cm}^{2}$ and $13.8 \mathrm{~J}$. A low fluence and short exposure time (10 minutes) were chosen to minimize any effects of PDT, allowing multiple treatment sessions in order to observe the time evolution of the field cancerization data in more detail. Exposure to the LED light protocol was performed on each hemiface while protecting the other hemiface from light. During application of the LED, the skin was kept cool with cold air (Siberian ${ }^{\circledR}$, Indústria Mecânica Fina) and cold water spray. Four PDT sessions, including applications of nano-ALA and MAL, Wood's illumination for image recording, and LED light treatment, were done in the three patients with a 3-4-week interval between sessions, depending on the patient's schedule for visiting the hospital service. Prior to all PDT sessions, the patients were clinically evaluated for both onset of new actinic keratoses and/or photodamaged areas, and the time evolution of the treated areas.

Fluorescence-sensitive imaging software was developed in order to analyze the time evolution of the as-treated areas in a quantitative manner using the protocol herein described, whether related to actinic keratosis or photodamage. In brief, this software loads the digitally recorded fluorescent areas under Wood's lamp illumination with the skills of sampling and using a new filter and using a new filter for colorimetric image segmentation based on red, green and blue (RGB) color space. The software evaluates the luminescent area within a region of interest, defined as the closed white polygon, as described later on in this paper. As mentioned above, the asdrawn polygon is based on anatomical aspects of the patient's face, and is roughly symmetrical. We choose the RGB color space for image processing due to its hardware-oriented nature that matches the way humans perceive colors, being sensitive to red, green, and blue components of visible light. ${ }^{39}$ In addition, we implemented a feature in the as-developed software which allows the user to map the edges of field cancerization simultaneously in two selected images, ie, the original and the processed one. This feature allowed us to compare the results obtained using color segmentation by software with that obtained by human operators using edge recognition patterns while analyzing the healthy and field cancerization regions of the patient's face. This was the approach used for evaluation of the quality of the segmentation process. A more detailed description of this software can be found in a recent patent filing. ${ }^{40}$ For each hemiface, the total calculated areas are plotted against the duration of treatment (ie, PDT sessions) and the observed time decay is compared. Emphasis is on interpretation of the observed differences in the time decay constants (half-life) of field cancerization, extent of the targeted area of field cancerization, and fractional reduction in the area of field cancerization in terms of photosensitizing nanoencapsulation versus non-nanoencapsulation.

\section{Results and discussion}

Figure 1A shows a typical transmission electron micrograph of the as-prepared nanoemulsion, highlighting the suspended nanocapsule, with the phospholipid-based shell pictured as a dark ring surrounding the light inner oily content. Figure 1B shows a diagram of the nanocapsule suspended within the nanoemulsion, revealing the phospholipid structure of the shell of the nanocapsule with the lipophilic tails of the molecules facing the oily interior and the polar heads facing outwards. Figure 1C shows the typical hydrodynamic size distribution of the 5-aminolevulinic acid-loaded nanoemulsion obtained by dynamic light scattering $(210 \mathrm{~nm})$, while Figure $1 \mathrm{D}$ shows the typical zeta potential distribution of the 5-aminolevulinic acid-loaded nanoemulsion obtained by electrophoretic measurement $(-51.4 \mathrm{mV})$.

Data on Table 1 shows the size increment of the ALAloaded nanocapsules (210.2 $\mathrm{nm}$ average diameter) compared with unloaded nanocapsules (180.0 nm average diameter), thus supporting the anchoring of ALA molecules onto the external surface of the nanocapsule. Further, the average $15 \mathrm{~nm}$ increment in the thickness of the nanocapsule shell supports the hypothesis of the onset of a well structured bond-based interfacial domain, which we believe confers high stability to the nanostructured end product. Furthermore, the average zeta potential data included in Table 1 shows a substantial reduction, from -40.3 to $-51.4 \mathrm{mV}$, as a result of ALA nanoencapsulation, representing a significant change to the external surface of the nanocapsule, believed to be due to anchoring of ALA molecules.

Following the protocol described earlier, Figure 2 shows a typical digital image of a fluorescent area on a patient's face while under Wood's lamp illumination. Although the skin itself has a fluorescence background, the protocol used in the present study allowed observation of both fluorescent and nonfluorescent areas, variations in these areas from patient to patient, and between MAL and nano-ALA photosensitizers. 

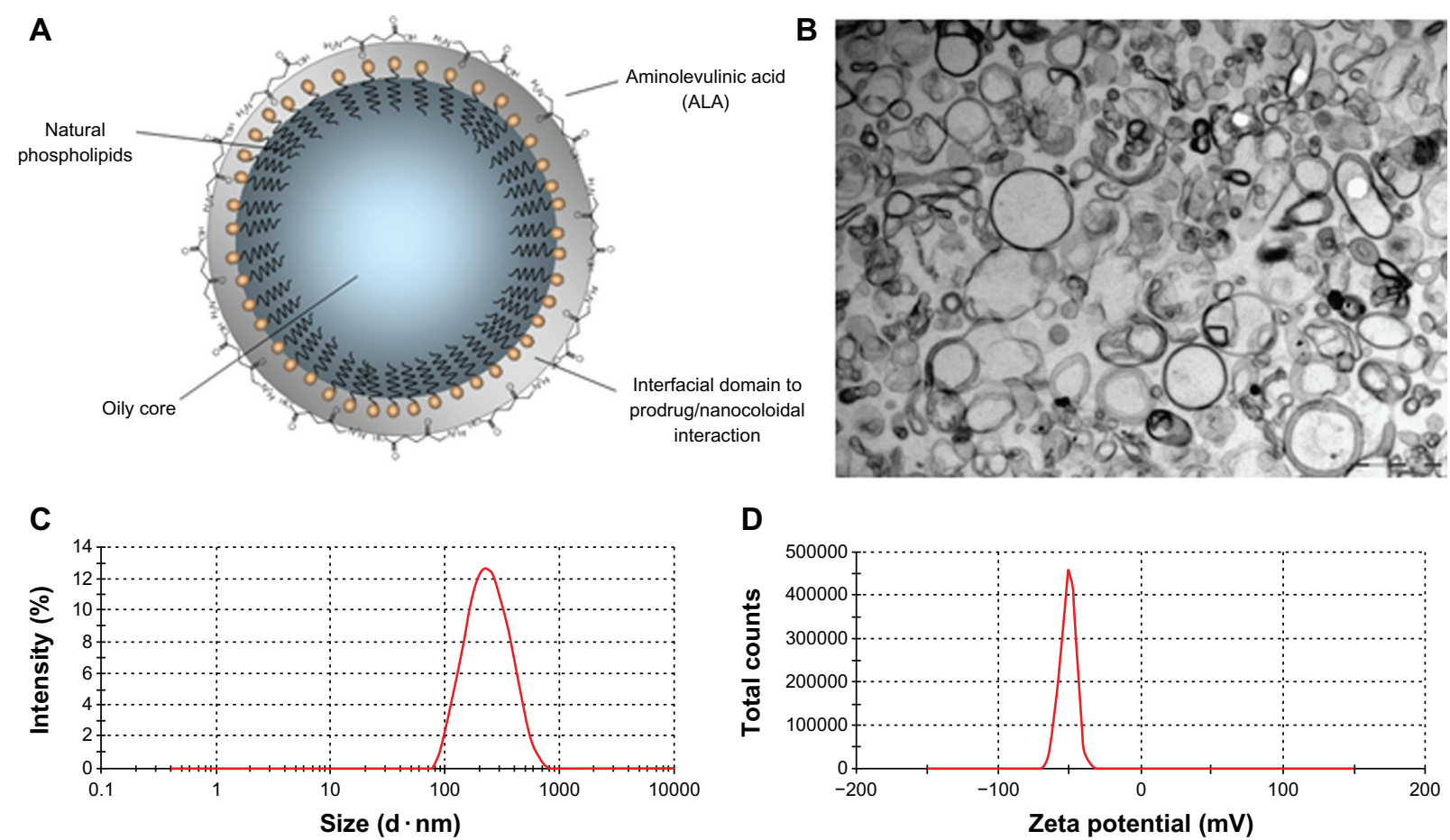

Figure I (A) Diagram representing the as-produced nanoemulsion, (B) typical transmission electron micrograph of the as-produced nanoemulsion, (C) typical hydrodynamic size distribution of the 5-aminolevulinic acid-loaded nanoemulsion obtained by dynamic light scattering, and (D) typical zeta potential distribution of the 5-aminolevulinic acid-loaded nanoemulsion obtained by electrophoretic measurement.

Notice the as-drawn polygon (solid white line) and the assessed inner luminescent area (red colored) in the left and right panels, respectively. Figure 3 shows the images of a typical time course (days) of treatment, as stated in the protocol for recording the pictures on both sides of the face under Wood's lamp illumination. At this point, we can report that the recorded luminescent area scales linearly with the extent of field cancerization, following the relationship observed between levels of protoporphyrin IX and clearance of lesions after photodynamic-MAL therapy. ${ }^{41}$

In order to carry out a quantitative study of the asdescribed PDT protocol for both treatment and evaluation of field cancerization associated with actinic keratosis, the time dependence of the luminescent areas recorded from all patients was collected and is shown in Figure 4, which

Table I Physicochemical parameters of nanocapsules loaded and not loaded with 5 -aminolevulinic acid

\begin{tabular}{lll}
\hline Sample & NC & NC-ALA \\
\hline Size $(\mathrm{nm})$ & 180.0 & 210.2 \\
Size dispersion & 0.08 & 0.16 \\
Zeta potential $(\mathrm{mV})$ & -40.3 & -51.4 \\
Solubility & Aqueous & Aqueous \\
Physicochemical stability (days) & 90 & 90 \\
PDT pathway mechanism & - & Type II $\left(\mathrm{CO}_{2}\right)$ \\
\hline
\end{tabular}

Abbreviations: NC, nanocapsules not loaded with 5-aminolevulinic acid; NC-ALA, nanocapsules loaded with 5-aminolevulinic acid; PDT, photodynamic therapy. represents the luminescent area normalized with respect to the recorded area. The recorded area is the area (inner) delimited by the solid white line polygon (see Figure 2). The normalization procedure takes into account the luminescent area of the very first PDT session for each patient and each hemiface. The number associated with the normalized area represents the number of pixels associated with the luminescent area over the number of pixels associated with the as-drawn polygon (solid white line). It should also be noted that one single exponential decay function was used for fitting the experimental data for both nano-ALA and MAL, as shown in Figure 4, regardless of the intraindividual aspects of the data analysis. Nevertheless, the field cancerization time decay constants for the two formulations were different, being about 62.5 and 49.5 days for nano-ALA and MAL, respectively. This translates into a field cancerization half-life, respectively, of about 43.3 and 34.3 days for nano-ALA and MAL. Although the difference in reduction of the half-life of the field cancerization area is about $26 \%$ longer for the nano-ALA formulation, the average field cancerization area targeted by nano-ALA is around $45 \%$ higher than the average area targeted by the MAL formulation. In addition, for the time window presented in Figure 4, the average reduction in field cancerization area using the nano-ALA formulation is around $10 \%$ greater 


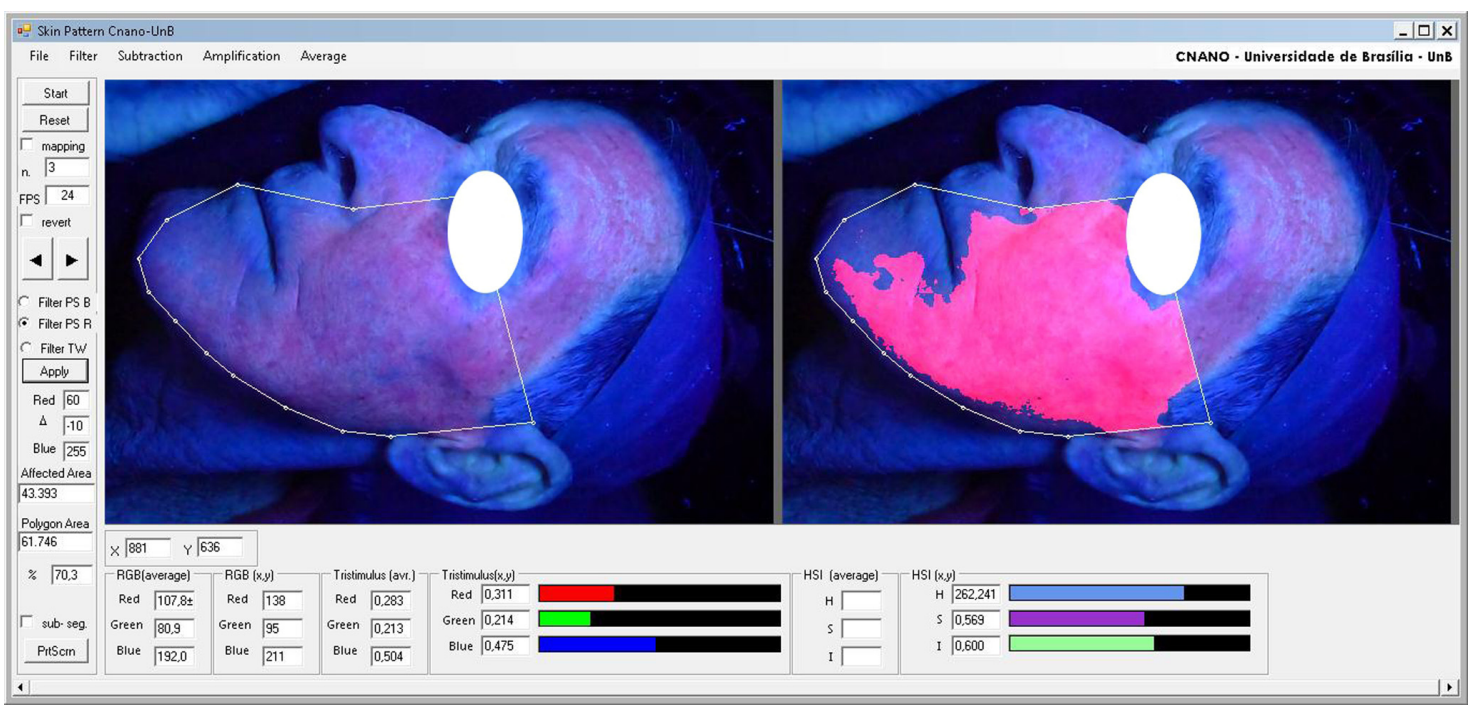

Figure 2 White polygon on both images defines the region of interest for color segmentation of field cancerization.

Notes: The left panel is the original image and the right panel is the treated image with the segmented area shown in red. Note that the software has a feature that allows the user to map the edges of field cancerization simultaneously in the two images in order to assess the quality of the segmentation process.

than the average reduction in field cancerization area using the MAL formulation. These findings have a wide range of fundamental and clinical implications.

From the clinical point of view, the largest field cancerization area targeted by the nano-ALA formulation (45\%) as well as the slightly higher field cancerization area reduction (10\%) represent an extremely valuable benefit in favor of ALA nanoencapsulation. However, the shortest reduction in half-life in field cancerization, apparently favoring the MAL formulation, needs to be clarified by more indepth analysis, as indicated below. To have clinical application, the balance between the benefits of one approach versus the other should be weighed on a case-to-case basis by the attending physician. From the fundamental point of view, a relevant aspect of our findings is the difference between the two formulations (nano-ALA and MAL) in the extent of targeted field cancerization after occluding the treated area for only 2 hours, when the typical occlusion time reported in the literature for free ALA is more

Right hand-side of the patient's face treated with MAL

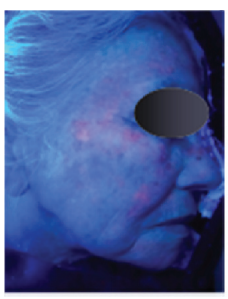

Day 0

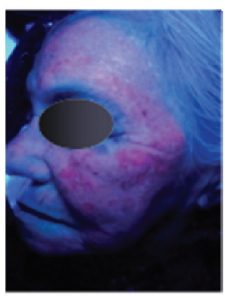

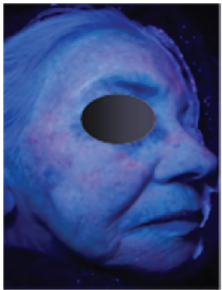

Day 28

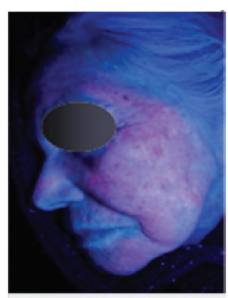

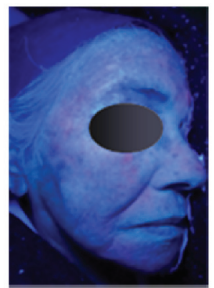

Day 49

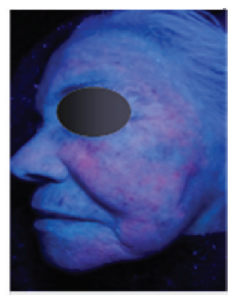

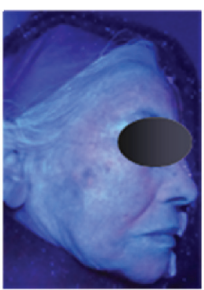

Day 70

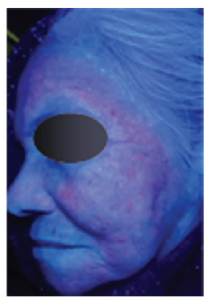

Left hand-side of the patient's face treated with nano-ALA

Figure 3 Sequence of images showing the time course of photodynamic therapy.

Note: The upper panel shows the right side of the patient's face (using MAL) and the lower panel shows the left side of the patient's face (treated with 5-aminolevulinic acid-loaded nanoemulsion). 


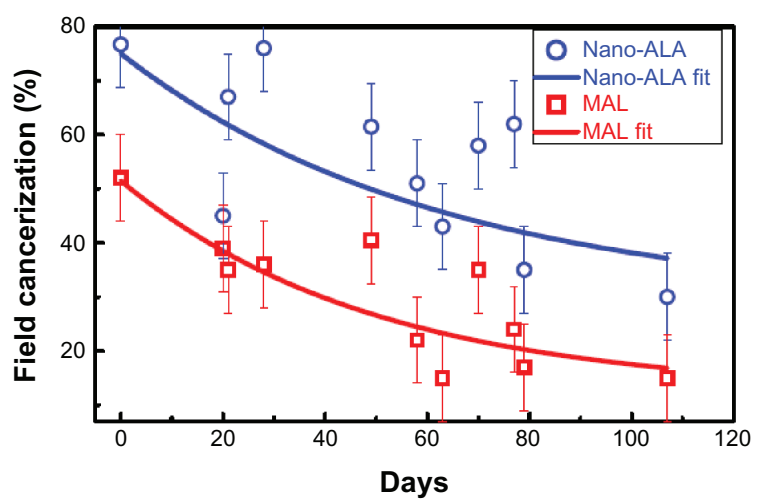

Figure 4 Time decay of field cancerization.

Notes: Symbols (open circles and squares) represent the experimental points and the solid lines going through the symbols represent the best curve-fitting according to time-dependent exponential decay. Symbols and the corresponding fitted solid lines are shown in blue for 5 -aminolevulinic acid-loaded nanoemulsion (open circles) and red for MAL (open squares).

than 4 hours. We found from analysis of the data shown in Figure 4 that nano-ALA targets a larger area than MAL. This is indeed a key difference between the two formulations and has to do with the higher efficiency of the nano-ALA formulation in targeting field cancerization, indicating one benefit of nanoencapsulation. We argue that this targeting efficiency is more likely due to the greater diffusion of the nano-ALA formulation into the skin. Indeed, the literature reports high skin diffusion rates associated with drug-loaded nanocapsules and the key role played by the structure of the phospholipid-based shell. ${ }^{42}$ Based on this information, we believe that both the highest field cancerization area reduction (10\%) and the longest reduction in half-life in field cancerization (about 26\%) are consequences of the greater efficacy of the nano-ALA formulation in targeting field cancerization.

\section{Conclusion}

In summary, this paper introduces a novel, inexpensive, and simple approach that allows quantification of field cancerization associated with actinic keratosis and skin photodamage. Although current techniques allow monitoring of protoporphyrin IX fluorescence after application of a prodrug, ${ }^{41}$ no quantitative approach is available. Thus, the present proposed strategy fills an important gap in the literature as well as in the clinic. The protocol introduced herein is based on the quantitative evaluation of the fluorescent area after topical application of a photosensitizing precursor, following occlusion by 2 hours before using Wood's lamp illumination. The fluorescent skin area was digitally collected and its extent quantitatively evaluated using color image segmentation software especially developed for this application. Further, the approach introduced in this paper was used to assess information about the efficacy of skin field cancerization treatment using PDT.
Two photosensitizing precursors, namely the commercial formulation of MAL (Metvix) and a new nanostructured formulation for 5-aminolevulinic acid (nano-ALA), were used and comparatively evaluated in the pilot trial reported here. Our approach to evaluation of quantitative skin field cancerization allowed us to assess the information regarding the time decay constants (half-life) associated with reduction of the recorded luminescent area for both MAL (half-life 34.3 days) and nanoALA (half-life 43.3 days). Further, our quantitative approach for probing skin field cancerization indicates that nano-ALA probes a $45 \%$ larger field cancerization area than MAL while using only 2 hours of occlusion. Finally, we found the reduction in area of skin field cancerization was $10 \%$ higher while using nano-ALA instead of MAL. Indeed, these findings have important implications, from fundamental aspects, eg, the changes in clinical response introduced by nanoencapsulation of a free drug, through to clinical aspects, eg, the feasibility of applying the approach presented here as a routine tool for detection, treatment, and follow-up of actinic keratosis and skin photodamage.

\section{Acknowledgments}

The authors acknowledge the financial support of the Brazilian agencies, Foundation for Research Support of the Federal District (FAPDF), Midwestern and Northen Network for RDI and Post-graduation in Nanobiotechnology/ Coordination of Improvement of Higher Education Personnel (Network CON-NANO/CAPES), National Institute of Science and Technology - Nanobiotechnology/ Ministry of Science, Technology and Innovation (INCT-Nano/MCTI), Foundation for Research Support of the State of São Paulo (FAPESP) (grant 08/53719-4 and post-doctoral grant support 09/15363-9), Deanship of Research and Graduate Studies University of Brasília (DPP-UnB), National Council for Scientific and Technological Development (CNPq) and Center of Nanoscience and Nanobiotechnology - University of Brasília (CNANO-UnB).

\section{Disclosure}

A patent relating to this work has been filed, ie, de Souza PEN, Morais PC software for colorimetric segmentation, ColorSeg Instituto Nacional de Propriedade Industrial, register number 12876-4, February 24, 2012. Otherwise the authors report no conflicts of interest in this work.

\section{References}

1. Braakhuis BJM, Tabor MP, Kummer JA, Leemans CR, Brakenhoff RH A genetic explanation of Slaughter's concept of field cancerization: evidence and clinical implications. Cancer Res. 2003;63:1727-1730. 
2. Califano J, van der Riet P, Westra W, et al. Genetic progression model for head and neck cancer: implications for field cancerization. Cancer Res. 1996;56:2488-2492.

3. Carlson JA, Scott D, Wharton J, Sell S. Incidental histopathologic patterns: possible evidence of "field cancerization" surrounding skin tumors. Am J Dermatopathol. 2001;23:4004-4006.

4. Jonason AS, Kunala S, Price GJ, et al. Frequent clones of p53-mutated keratinocytes in normal human skin. Proc Natl Acad Sci USA. 1996;93: 14025-14029.

5. Williams C, Ponten F, Ahmadian A, et al. Clones of normal keratinocytes and a variety of simultaneously present epidermal neoplastic lesions contain a multitude of $\mathrm{p} 53$ gene mutations in a xeroderma pigmentosum patient. Cancer Res. 1998;58:2449-2455.

6. Rubin H. Fields and field cancerization: the preneoplastic origins of cancer. Bioessays. 2011;33:224-231.

7. Brennan JA, Mao L, Hruban RH, Boyle JO, Koch WM, Sidransky D. Molecular assessment of histopathological staging in squamous cell carcinoma of the head and neck. N Engl J Med. 1995;332:429-435.

8. Tschen EH, Wong DS, Pariser DM, Dunlap FE, Houlihan A, Ferdon MB. Photodynamic therapy using aminolaevulinic acid for patients with nonhyperkeratotic actinic keratoses of the face and scalp: Phase IV multicentre clinical trial with 12-month follow up. $\mathrm{Br} J$ Dermatol. 2006;155:1262-1269.

9. Brash DE, Ponten J. Skin precancer. Cancer Surv. 1998;32:69-113.

10. Padilla RS, Sebastian S, Jiang Z, Nindl I, Larson R. Gene expression patterns of normal human skin, actinic keratosis, and squamous cell carcinoma: a spectrum of disease progression. Arch Dermatol. 2010; 146:288-293.

11. Röwert-Huber J, Patel M, Forschner T, et al. Actinic keratosis is an early in situ squamous cell carcinoma: a proposal for reclassification. Br J Dermatol. 2007;156:Suppl 3:8-12.

12. Ackerman AB. Solar keratosis is squamous cell carcinoma. Arch Dermatol. 2003;139:1216-1217.

13. Heaphy MR Jr, Ackerman AB. The nature of solar keratosis: a critical review in historical perspective. J Am Acad Dermatol. 2000;43: 138-150.

14. Glogau RG. The risk of progression to invasive disease. J Am Acad Dermatol. 2000;42:23-24.

15. Ramsay HM, Fryer AA, Reece S, Smith AG, Harden PN. Clinical risk factors associated with non-melanoma skin cancer in renal transplant recipients. Am J Kidney Dis. 2000;36:167-176.

16. Braathen LR, Szeimies RM, Basset-Seguin N, et al. Guidelines on the use of photodynamic therapy for nonmelanoma skin cancer: an international consensus. J Am Acad Dermatol. 2007;56:125-143.

17. Stockfleth E, Kerl H. Guidelines for the management of actinic keratoses. Eur J Dermatol. 2006;16:599-606.

18. De Berker D, McGregor J, Hughes B. Guidelines for the management of actinic keratoses. Br J Dermatol. 2007;156:222-230.

19. Babilas P, Schreml S, Landthaler M, Szeimies RM. Photodynamic therapy in dermatology: state-of-the-art. Photodermatol Photoimmunol Photomed. 2010;26:118-132.

20. Piacquadio DJ, Chen DM, Farber HF, et al. Photodynamic therapy with aminolevulinic acid topical solution and visible blue light in the treatment of multiple actinic keratoses of the face and scalp: investigatorblinded, phase 3, multicenter trials. Arch Dermatol. 2004;140:41-46.

21. Varma S, Wilson H, Kurwa HA, et al. Bowen's disease, solar keratoses and superficial basal cell carcinomas treated by photodynamic therapy using a large-field incoherent light source. Br J Dermatol. 2001;144: 567-574.

22. Braathen LR, Morton CA, Basset-Seguin N, et al. Photodynamic therapy for skin field cancerization: an international consensus. International Society for Photodynamic Therapy in Dermatology. JEur Acad Dermatol Venereol. 2012;26:1063-1066.

23. Henderson BW, Dougherty TJ. How does photodynamic therapy work? Photochem Photobiol. 1992;55:145-157.
24. Angel-Petersen E, Sørensen R, Warloe T, et al. Porphyrin formation in actinic keratosis and basal cell carcinoma after topical application of methyl-5- aminolevulinate. J Invest Dermatol. 2006;126:265-271.

25. Gardlo K, Ruzicka T. Metvix (PhotoCure). Curr Opin Investig Drugs. 2002;3:1672-1678.

26. Peng Q, Soler AM, Warloe T, Nesland JM, Giercksky KE. Selective distribution of porphyrins in skin thick basal cell carcinoma after topical application of methyl 5-aminolevulinate. JPhotochem Photobiol B. 2001;62:140-145.

27. Fritsch C, Homey B, Stahl W, Lehmann P, Ruzicka T, Sies H. Preferential relative porphyrin enrichment in solar keratoses upon topical application of delta-aminolevulinic acid methyl-esther. Photochem Photobiol. 1998;68:218-221.

28. Szeimies R, Karrer S, Radakovic-Fijan S, et al. Photodynamic therapy using topical methyl 5-aminolevulinate compared with cryotherapy for actinic keratosis: a prospective, randomized study. J Am Acad Dermatol. 2002;47:258-262.

29. Morton CA, Brown SB, Collins S, et al. Guidelines for topical photodynamic therapy: report of a workshop of the British Photodermatology Group. Br J Dermatol. 2002;146:552-567.

30. Pariser DM, Lowe NJ, Stewart DM, et al. Photodynamic therapy with topical methyl aminolevulinate for actinic keratosis: results of a prospective randomized multicenter trial. J Am Acad Dermatol. 2003;48:227-232.

31. Di Venosa G, Hermida L, Batlle A, et al. Characterization of liposomes containing aminolevulinic acid and derived esters. J Photochem Photobiol B Biol. 2008;92:1-9.

32. Moloney FJ, Collins P. Randomized, double-blind, prospective study to compare topical 5-aminolaevulinic acid methylester with topical 5-aminolaevulinic acid photodynamic therapy for extensive scalp actinic keratosis. Br J Dermatol. 2007;157:87-91.

33. Washbrook R, Riley PA. Comparison of delta-aminolaevulinic acid and its methyl ester as an inducer of porphyrin synthesis in cultured cells. Br J Cancer. 1997;75:1417-1420.

34. Casas A, Fukuda H, Di Venosa G, Batlle A. Photo-sensitization and mechanism of cytotoxicity induced by the use of ALA derivatives in photodynamic therapy. Br J Cancer. 2001;85:279-284.

35. Krammer B, Verwanger T. Photodynamic therapy with aminolevulinic acid or its methyl ester: which one is superior? Med Laser Appl. 2009;24: 221-226.

36. Cheng HD, Jiang XH, Sun Y, Wang J. Color image segmentation: advances and prospects. Pattern Recognit. 2001;34:2259-2281.

37. Primo, FL, Reis MBC, Porcionatto MA, Tedesco AC. In vitro evaluation of chloroaluminum phthalocyanine nanoemulsion and low-level laser therapy on human skin dermal equivalents and bone marrow mesenchymal stem cells. Curr Med Chem. 2011;18:3376-3381.

38. Szeimies RM, Matheson RT, Davis SA, et al. Topical methyl aminolevulinate photodynamic therapy using red light-emitting diode light for multiple actinic keratoses: a randomized study. Dermatol Surg. 2009;35:586-592.

39. Gonzales RC, Richard E. Woods Digital Image Processing, 3rd ed. Upper Saddle River, NJ: Prentice Hall; 2008.

40. De Souza PEN, Morais PC. Software for colorimetric segmentation ColorSeg. Instituto Nacional de Propriedade Industrial - INPI, register n 12876-4; February 24, 2012.

41. Tyrrell JS, Campbell SM, Curnow A. The relationship between protoporphyrin ix photobleaching during real-time dermatological methylaminolevulinate photodynamic therapy (MAL-PDT) and subsequent clinical outcome. Lasers Surg Med. 2010;42:613-619.

42. Primo FL, Michieleto L, Rodrigues MAM, et al. Magnetic nanoemulsions as drug delivery system for Foscan ${ }^{\circledR}$ : Skin permeation and retention in vitro assays for topical application in photodynamic therapy (PDT) of skin cancer. J Magn Magn Mater. 2007;311:354-357. 


\section{Publish your work in this journal}

Clinical, Cosmetic and Investigational Dermatology is an international, peer-reviewed, open access, online journal that focuses on the latest clinical and experimental research in all aspects of skin disease and cosmetic interventions. All areas of dermatology will be covered; contributions will be welcomed from all clinicians and basic science researchers globally. This journal is indexed on CAS. The manuscript management system is completely online and includes a very quick and fair peer-review system, which is all easy to use. Visit http://www.dovepress.com/testimonials.php to read real quotes from published authors.

Submit your manuscript here: http://www.dovepress.com/clinical-cosmetic-and-investigational-dermatology-journal 